Volumen: 30 Fecha de recibido: 12/1/2020 Fecha de publicación: Julio-Diciembre de 2020

Año: 2020 Fecha de aceptado: 24/2/2020 Correo: revista.sciencia@up.ac.pa

Numero: 2 Número de páginas: 29-41 URL: https://revistas.up.ac.pa/index.php/scientia

\title{
ABUNDANCIA Y DIVERSIDAD DE CARACOLES (MOLLUSCA: GASTROPODA) EN LAS PLAYAS NOMBRE DE DIOS, PALENQUE Y VIENTO FRIO, PROVINCIA DE COLÓN
}

\author{
Luis Sánchez ${ }^{1}$, Mario González ${ }^{1}$, Berta Liz Peña ${ }^{2}$, Jorge Gutierrez ${ }^{2}$ \\ 1-Universidad de Panamá, Centro Regional Universitario de Colón, Panamá, Panamá \\ luis.sanchezo@up.ac.pa, mario.gonzalezt@up.ac.pa \\ 2-Universidad de Panamá, Facultad de Ciencias Naturales, Exactas y Tecnología, Panamá, Panamá \\ jlgutiz@yahoo.es, liznerita_2625@hotmail.com
}

\section{RESUMEN}

Esta investigación se realizó en las playas Nombre de Dios, Viento Frio, y Palenque, que están ubicadas en el Distrito de Santa Isabel, Costa Arriba de la Provincia de Colón. Las muestras se colectaron de forma manual, luego fueron colocadas en envases plásticos rotulados. para luego ser trasladadas al laboratorio de la Escuela de Biología del Centro Regional Universitario de Colón donde se limpiaron y luego se caracterizaron, a nivel de especie Además de un análisis de varianza, y se calcularon índices de diversidad como al alfa de Williams, de dominancia como el BergerParker o el de Margaleff, Se encontró que no hubo diferencia de abundancia significativa $(\mathrm{F}=0.5984 ; p>0.5552)$ entre las playas. Los índices de diversidad indicaron que hubo una gran equidad en la distribución de las especies en las playas, siendo Viento Frío la de mayor equidad con 6,92; la dominancia fue baja, el mayor valor se obtuvo en Viento Frío, con 0,33. Para el índice de Margaleff, el mayor valor fue en Nombre de Dios a 18,863. Se colectaron 134 individuos pertenecientes a 28 especies, 20 géneros, de los cuales la especie más abundante fue Littorina ziczac (Gmelin, 1791), con 24 individuos, Tanto Nerita versicolor (Gmelin, 1791) con 19, y Nerita tesellata (Gmelin, 1791) con 12, mientras que Certhiopsis greeni (C. B. Adams, 1838) con 15. También se reportaron 13 especies con un solo individuo: Arene variabilis (Dall, 1889), Batillaria mínima (Gmelin, 1791), Cerithium atratum (Born,1778), Cymatium parthemopeum (von Salis, 1793), Leurosyrinx verrillii (Dall, 1881), Mitra barbadensis (Gmelin, 1791), Ocenebra muricoides (C. B Adams, 1845), Polinices hepaticus (Röding, 1869), Purpura patula (Linnaeaus, 1758), Tachypollia didyma (Schwengel, 1943), Terebra cinerea Born, 1778 y Turbinella angulata (Lightfoot, 1786).

PALABRAS CLAVES: Diversidad de Especies, Eurihalina, Estenohalinas, Tipos de Sustrato 


\begin{abstract}
This investigation was carried out on the beaches Nombre de Dios, Viento Frio, and Palenque, which are in the District of Santa Isabel, Costa Arriba of the Province of Colón. The samples were collected manually, then placed in labeled plastic containers. to be transferred to the laboratory of the School of Biology of the Regional University Center of Colon where they were cleaned and then characterized, at the species level In addition to an analysis of variance, and diversity indexes were calculated as Williams alpha, dominance such as the Berger-Parker or the Margaleff, it was found that there was no significant abundance difference $(\mathrm{F}=0.5984 ; p>0.5552)$ between the beaches. Diversity indices indicated that there was great equity in the distribution of species on the beaches, with Viento Frio being the most equitable with 6.92; dominance was low, the highest value was obtained in Cold Wind, with 0.33. For Margaleff's index, the highest value was in Nombre de Dios to 18,863. We collected 134 individuals belonging to 28 species, 20 genera, of which the most abundant species was Littorina ziczac (Gmelin, 1791), with 24 individuals, both Nerita versicolor (Gmelin, 1791) with 19, and Nerita tesellata (Gmelin, 1791). ) with 12, while Certhiopsis greeni (CB Adams, 1838) with 15. Also 13 species were reported with a single individual: Arene variabilis (Dall, 1889), Batillaria minima (Gmelin, 1791), Cerithium atratum (Born, 1778) , Cymatium parthemopeum (von Salis, 1793), Leurosyrinx verrillii (Dall, 1881), Mitra barbadensis (Gmelin, 1791), Ocenebra muricoides (C. Adams, 1845), Polynices hepaticus (Röding, 1869), Purpura patula (Linnaeaus, 1758), Tachypollia didyma (Schwengel, 1943), Terebra cinerea Born, 1778 and Turbinella angulata (Lightfoot, 1786).
\end{abstract}

KEY WORDS: Diversity of Species, Eurihalins, Stenohalins, Substrate Types

\title{
INTRODUCCIÓN
}

Los ecosistemas acuáticos a nivel mundial son los más extensos y de mayor importancia económica. Son vitales por su rica biodiversidad de especies marina en especial de los caracoles (Mollusca: Gastropoda). Colón cuenta con amplias playas rodeadas de arrecifes de coral con abundancia de moluscos que consideramos prioritarios en la economía, el turismo y nuestro ambiente, que debemos conservar. Ante el avance del desarrollo industrial, el ecoturismo y la urbanización de las tierras aledañas a nuestras costas se ha incrementado la destrucción y contaminación de los hábitats marinos. (Barrientos, 2003) Lo que afecta las especies de Mollusca: Gastropoda. El estudio de los caracoles (Mollusca: Gastropoda) en cuanto a su biodiversidad, 
estado de conservación y su distribución geográfica permitirá documentar la riqueza de especies y establecer una línea base para posteriores estudios de conservación y ecología de la diversidad de este grupo natural. (Nieto-Bernal et al, 2013; Rodríguez et al, 2003)

Los moluscos constituyen uno de los phylla más interesantes de estudiar ya que son buenos bioindicadores que permiten conocer cambios ocurridos en el medio ambiente como respuesta a intervenciones humanas, ya que por medio de ellos nos damos cuenta de la calidad del agua y de la contaminación. Además, son proveedores de excelentes modelos para estudiar patrones de biodiversidad y también procesos evolutivos. Constituyen el grupo más diverso en el reino animal después de los artrópodos. Existen aproximadamente más de 75000 especies vivas y 15000 fósiles descritas. Se pueden encontrar en casi todo tipo de ambientes (inclusive desiertos), pero mayoritariamente en aguas saladas o dulces, aunque unos pocos han logrado colonizar el medio terrestre, siendo los Gastropoda el único grupo de moluscos con representantes en tierra firme. Por lo tanto, los moluscos están ampliamente distribuidos a lo largo del planeta debido a sus adaptaciones morfológicas que les han permitido colonizar estos distintos tipos de ambientes. (Solís, 2004)

En Panamá contamos con extensas costas en ambos océanos y la provincia de Colón cuenta con cinco distritos: Colón, Chagres, Donoso, Portobelo y Santa Isabel; y 40 corregimientos. Cada corregimiento cuenta con amplias playas en el mar Caribe.

\section{MATERIALES Y MÉTODOS}

Esta investigación se hizo en tres playas de la Costa Arriba de la Provincia de Colón, Nombre de Dios cuyas coordenadas geográficas son: $9^{\circ} 35^{\prime} 0,20^{\prime} \mathrm{N}$ por $79^{\circ} 28^{\prime} 13,05^{\prime}$ O, Viento Frio 9³4'0,20” N por 79²8'13,05” O., y Palenque $9^{\circ} 34^{\prime} 23,13^{\prime \prime}$ N por 79²1'31,56” O., ubicadas en 
el Distrito de Santa Isabel. Estas playas tienen en común el que contienen grandes extensiones de coral, aunque la difieren en extensión y en forma. Las muestras se colectaron de forma manual, luego fueron colocadas en envases plásticos rotulados con la siguiente información: fecha y lugar de colecta, tipo de sustrato y condiciones meteorológicas reinantes. para luego ser trasladadas al laboratorio E 1.3 de la Escuela de Biología del Centro Regional Universitario de Colón, donde se limpiaron y luego se caracterizaron, a nivel de especie, con la ayuda de claves taxonómicas y otras literaturas especializadas disponibles (Abbot, 1955; Abbot, 1959; Abbot, 1964; Abbot, 1974; Caicedo, 1984; Caicedo, 1989; Dance, 2002; García, 2010; García, 2011; Keen, 1971, Kiel, 2001; Malaquias y Reid, 2008; Spencer et al, 2007). En la medida que los muestreos avanzaban se construyó una colección de referencia, que luego fue llevada al Museo de Malacología y una base de datos. A esta data se le aplicaron análisis descriptivos como media, desviación estándar y coeficiente de variación y se confeccionaron graficas de pastel y barras, Además de un análisis de varianza, y se calcularon índices de diversidad como al alfa de Williams, de dominancia como el Berger-Parker o el de Margaleff, con el fin de estimar la abundancia y diversidad de este grupo en las playas mencionadas. (Moreno, 2001; Andrión y Danzic, 2010; González y Navarro, 1995) Los cálculos se realizaron con los programas computarizados STATISTICA 7.0 (Statsoft Inc., 2004).

La diversidad se calculó mediante índices de diversidad: Alfa de Williams, Berges-Parker y Margaleff. utilizando los programas Bio-Diversity Pro. 2.0 (McAleence, et. al) y PAST (Hammer, Ø. and Harper, R., 2001).

\section{RESULTADOS Y DISCUSIÓN}

Al comparar la abundancia de las playas se encontró que en la playa de Palenque se encontró $40 \%$ de la colecta ,seguido de Nombre de Dios con el 39\% y Viento Frío con el 21\% (Ver figura 1), No 
hay diferencia de abundancia no es significativa ( $\mathrm{F}=0.5984 ; \mathrm{p}>0.5552)$, lo que concuerda con lo encontrado en la playas de Piña, donde la falta de diferencia entre los muestreos pudo deberse a la condiciones ambientales reinantes (Acosta y Lima, 1997; González et al, 2011; González et al, 2018; González et al, 2019). Tal vez porque la estructura del sustrato de estas tres playas es bastante similar, conformado principalmente por planchas de coral y arenas (Aguilar et al, 2010; Miloslavich, y Huck 2009), con praderas de hierbas marinas del género Thalassia. (Figura 1) (Capelo y Buitrago, 1998; Correa-Sandobal y J. Rodriguez; 2013; González et al. 2018; Kohn, 1970)

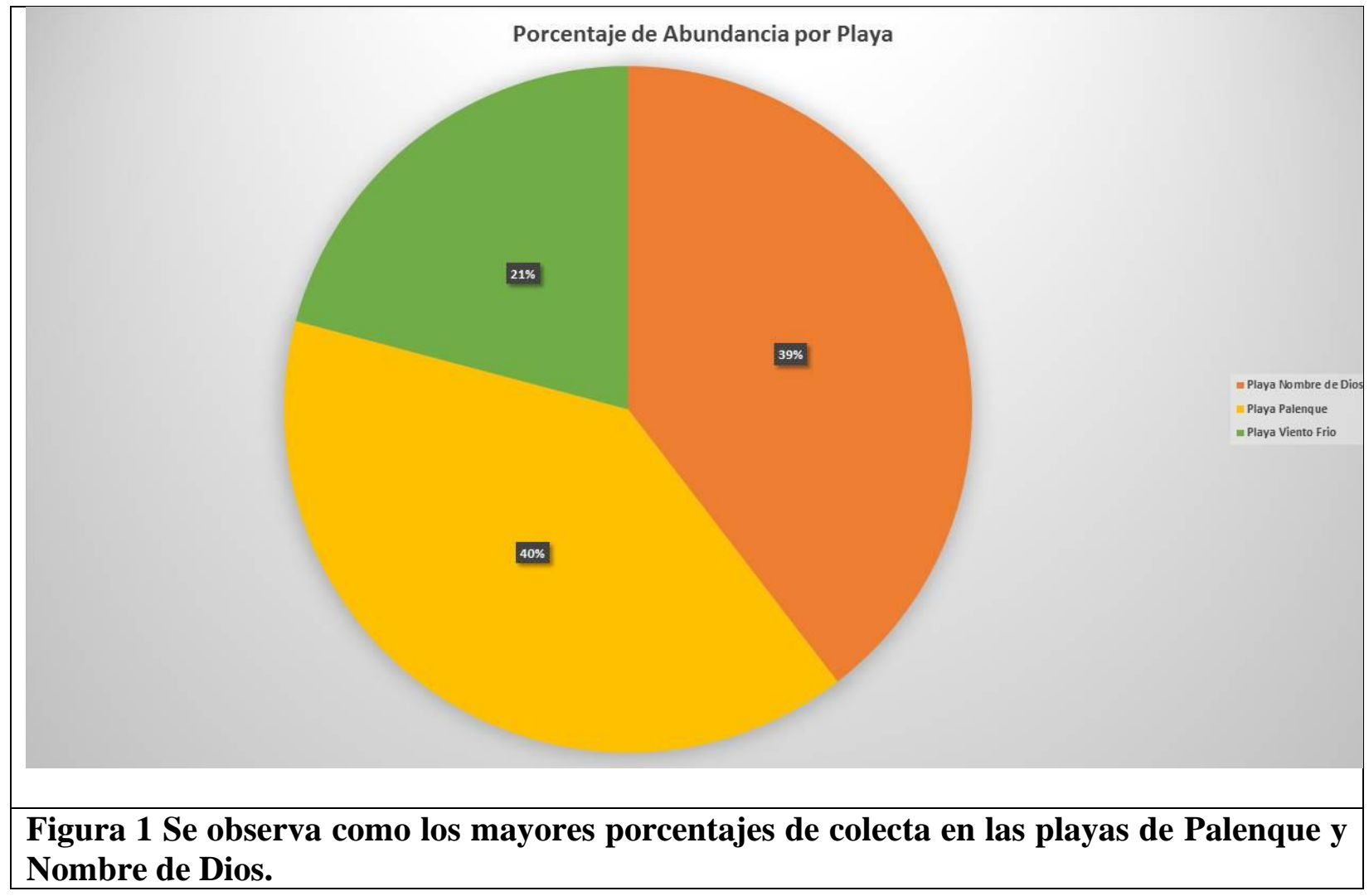

Los índices de diversidad Alfa de Williams y de dominancia Berger-Parker indicaron que hubo una gran equidad en la distribución de las especies en las playas, siendo Viento Frío la de mayor 
equidad con 6,92 y Nombre de Dios con 6,06; la dominancia fue baja, la mayor se obtuvo en Viento Frío, con 0,33 y la menor en Nombre de Dios con 2,55. El índice de Margaleff, indicó valores entre 15,51 en Nombre de Dios a 18,86 en Viento Frío. Al observar los valores de los índices en estas playas puede notarse que Viento Frío tiene los mayores valores, mientras que Nombre de Dios los menores, esto pudo deberse principalmente a la forma de estas playas, mientras que La playa de Viento Frío es mayoritariamente coralina y con forma lineal. La playa de Nombre de Dios tiene la forma de una herradura que encierra una bahía compuesta por un sustrato de arenas y praderas de hierbas marinas, y los arrecifes coralinos se encuentran mayoritariamente en la entrada de esta, haciéndole frente al oleaje. Sin embargo, estas diferencias no son grandes al extremo de que los análisis las consideran como no significativas, lo que implicaría que son más un producto de la casualidad (Castillo-Rodríguez, 2014; De La Rosa, 1998; Kohn, 1970; Ferney y Blanco, 2012; González y Navarro, 1995; Ríos, 2004).

\begin{tabular}{|c|c|c|}
\hline Familia & Género & Especie \\
\hline Areneidae & Arene & Arene variabilis(Dall, 1889) \\
\hline Batillariidae & Batillaria & Batillaria minima(Gmelin, 1791) \\
\hline Buccinidae & Engina & Engina turbinella (Kiener, 1835) \\
\hline Calliostomatidae & Leurosyrinx & Leurosyrinx verrillii(Dall, 1881) \\
\hline Cassidae & Cypraecassis & Cypraecassis testiculus(Linnaeaus, 1758) \\
\hline \multirow[b]{5}{*}{ Cerithidae } & \multirow[b]{5}{*}{ Cerithium } & Cerithium atratum(Born,1778) \\
\hline & & Cerithium eburneumBrugiére, 1792 \\
\hline & & Cerithium litteratum(Born, 1778) \\
\hline & & Cerithium lutosum Menke, 1828 \\
\hline & & Cerithium muscarumSay,1832 \\
\hline Cerithiopsidae & Cerithiopsis & Cerithiopsis greeni(C. B. Adams, 1838) \\
\hline \multirow[b]{2}{*}{ Littorinidae } & Littorina & Littorina ziczac (Gmelin, 1791) \\
\hline & Tectarius & Tectarius muricantus (Linnaeaus, 1758) \\
\hline Mitridae & Mitra & Mitra barbadensis (Gmelin, 1791) \\
\hline \multirow[b]{4}{*}{ Muricidae } & Ocenebra & Ocenebra muricoides (C. B Adams, 1845) \\
\hline & Purpura & Purpura patula (Linnaeaus, 1758) \\
\hline & \multirow[b]{2}{*}{ Thais } & Thais deltoidea (Lammark,1822) \\
\hline & & Thais haemastoma (Lammark,1822) \\
\hline
\end{tabular}




\begin{tabular}{|l|l|l|} 
& & Thais rustica (Lammark,1822) \\
\hline Naticidae & Polinices & Polinices hepaticus (Röding, 1869) \\
\hline \multirow{4}{*}{ Neritidae } & \multirow{4}{*}{ Nerita } & Nerita fulgurans (Gmelin, 1791) \\
\cline { 3 - 3 } & Nerita versicolor (Gmelin, 1791) \\
\cline { 3 - 3 } Planaxidae & Planaxis & Planaxis nucleus (Brugière, 1789) \\
\hline Ranellidae & Cymatium & Cymatium parthemopeum (von Salis, 1793) \\
\hline Terebridae & Terebra & Terebra cinerea Born, 1778 \\
\hline Turbinellidae & Turbinella & Turbinella angulata (Lightfoot, 1786) \\
\hline
\end{tabular}

Se colectaron 134 individuos pertenecientes a 28 especies, 20 géneros, de los cuales la especie más abundante fue Littorina ziczac (Gmelin, 1791), con 24 individuos, lo que es importante señalar ya que se trata de una especie estenohalina, que habita en el rompiente de las olas, por lo que no se le encuentra más allá y que no resiste largos periodos de tiempo descubierta por el agua de mar. (Bandel, 1974; Beltran, 1985) La mayor cantidad se colecto en Nombre de Dios con 14, debido principalmente a las aguas calmas de la bahía, que permiten que estos delicados organismos prosperen en las orillas cercanas a las playas a diferencia de los ocurrido en las playas de Piña donde la especie más abundante fue la Purpura patula.(Linnaeaus, 1758) que es un depredador (González et al, 2019; Navarrete, 1994) y en la playa la Escucha que fue Nerita fulgurans (Gmelin, 1791) (Qin, 1995; Blanco y Scatena, 2005; González et al, 2011; González et. al, 2018; Peña, 2000). Estas diferencias pueden deberse a la diferencia en posición geográfica de estas playas y la misma forma del istmo de Panamá, en donde Las playas de Nombre de Dios, Palenque y Viento Frío se encuentran en la parte más alta de la "S" acostada, mientras que las Playas de La Escucha y Piña se encuentra en la bajada de la "S", lo que expondría a estas playas a distintas corrientes que cambiarían la composición química de las aguas, la disposición de nutrientes, así como la turbiedad de las aguas, eso sin contar que entre las playas de La Escucha y Piña, existe una barrera de agua dulce artificial provenientes del canal de Panamá, la cual altera aún más todos los componentes 
químicos de las aguas, lo que se muestra en la abundancia y diversidad de especies presentes en estas playas. (Hurtado et al,2007) Tanto así que en la playa de Piña que se encuentra al oeste de la entrada del canal de Panamá, y su especie de caracol es un carnívoro, mientras que, en las playas de La Escucha, Nombre de Dios, Palenque y Viento Frío, que se encuentran al este de la entra del canal de Panamá, las especies más abundantes sin herbívoros.

Tanto Nerita versicolor (Gmelin, 1791) con 19, y Nerita tesellata (Gmelin, 1791) con 12, son especies eurihalinas, que pueden resistir grandes cantidades de tiempo alejada del agua marina se (Qin, 1995; Blanco y Scatena, 2005; Kiat y Clements, 2008; Mazenett-Gutierrez et al, 2012), por lo que su presencia en la muestra es casi segura, mientras que Certhiopsis greeni (C. B. Adams, 1838) con 15 ,es una especie que habita en las praderas de hierbas marinas, que encuentran más allá del nivel mínimo que baja la marea, lo que hace muy posible que su captura fuera accidental. (Rasmussen et al, 2005; De La Rosa, 1998; Castillo-Rodríguez, 2014; Capelo y Buitrago, 1998) También se reportaron 13 especies con un solo individuo: Arene variabilis (Dall, 1889), Batillaria mínima (Gmelin, 1791), Cerithium atratum(Born,1778), Cymatium parthemopeum (von Salis, 1793), Leurosyrinx verrillii (Dall, 1881), Mitra barbadensis (Gmelin, 1791), Ocenebra muricoides (C. B Adams, 1845), Polinices hepaticus (Röding, 1869), Purpura patula (Linnaeaus, 1758) quien es un depredador (Navarrete, 1994), Tachypollia didyma (Schwengel, 1943), Terebra cinerea Born, 1778 y Turbinella angulata (Lightfoot, 1786). Es posible que los individuos pertenecientes a estas especies no habiten normalmente los lugares donde se realizaron las colectas por lo que su captura puede considerarse como accidental. (Figura 2). En la playa de Piña también se encontraron individuos del género Cerithium, además de otras especies de las cuales solo había un individuo, posiblemente estos individuos eran arrastrados por la marea desde otros sustratos bajo el mar (González et. al, 2019) 


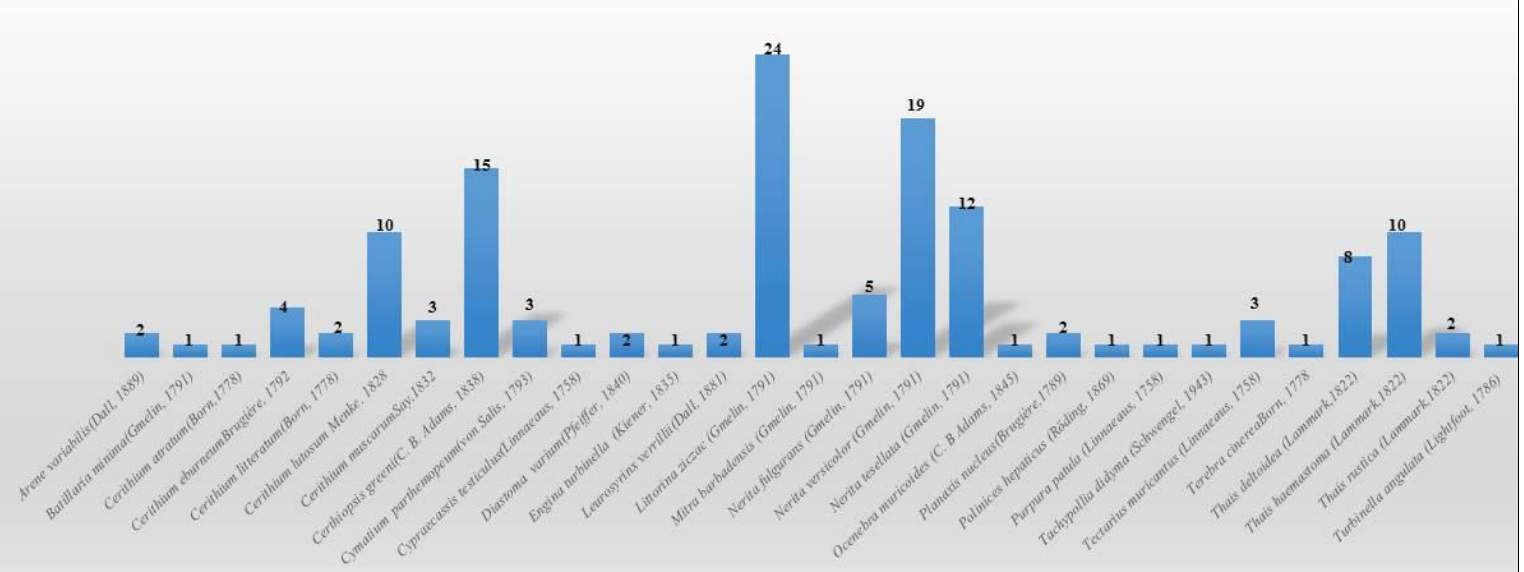

Figura 2 Puede destacarse la abundancia de Littorina ziczac y Nerita versicolor sobre las demás especies colectadas.

\section{CONCLUSIÓN}

La riqueza de especies fue de 28 especies de las cual la Littorina ziczac (Gmelin, 1791), fue la más abundante con 24 individuos, seguida de la Nerita versicolor (Gmelin, 1791) con 19, y Nerita tesellata (Gmelin, 1791) con 12, siendo importante el mencionar que al comparar la riqueza de especies y la abundancia de individuos en las playas donde se hizo este estudio, dio como resultado que no existía diferencia significativa entre estas playas.

\section{AGRADECIMIENTO}

Extendemos nuestro agradecimiento a Licdo. Darío Córdoba técnico del museo de Malacología de la Escuela de Biología de la Universidad de Panamá Campus "Octavio Méndez Pereira", a los estudiantes de la escuela de Biología del Centro Regional Universitario de Colón, quienes nos ayudaron en las colectas de campo, el etiquetado, traslado, y limpieza de las muestras para su posterior identificación. Al profesor Juan F. Macías director del Centro Regional Universitario de 
Colón por su ayuda al facilitarnos el bus del centro con su conductor para poder desarrollar las giras de campo. Al señor Alexis Deglas conductor del bus del Centro Regional Universitario de Colón. Al alcalde de Santa Isabel profesor Boris Góndola por todas sus atenciones y facilidades dadas para los trabajos en las playas. Los representantes de cada una de las comunidades, Nombre de Dios, Palenque y Viento Frío por todo su apoyo en la investigación.

\section{REFERENCIAS}

Abbott, R. T. (1955). American Seashells. Van Nostrand Company, firt edition, New York. U.S.A.

Abbot, R. (1959), American Seashell. D. Van Nostrod Company. New York. U.S.A.

Abbott, R. (1964) Littorina ziczac Gmelin and Littorina lineolate Orbigny. Nautilus 78, 65-66.

Abbot, R. (1974) American Seashell. D. Van Nostrod Company. New York. U.S.A.

Acosta, B. y D. Lima (1997) Biodiversidad, Abundancia y Distribución de Gasterópodos en la Bahía de Panamá. (Tesis de Licenciatura) Universidad de Panamá. Panamá.

Aguilar, T.; B. Acevedo y A. Ulloa (2010) Paleontología de una Sección del Río Corredores. Formación Curré Mioceno. Costa Rica. Rev. Geol. Amér. Central 42, 43-75.

Andrión, V. y A. Danzic (2010) Composición de Especies de Gastrópodas en la Playa "La Escucha" del Distrito de Portobelo, Provincia de Colón. (Tesis de Licenciatura) Universidad de Panamá. Panamá.

Bandel, K, (1974) Studies on Littorinidae from the Atlantic. Veliger. 17, 92-114.

Barrientos, Z. (2003) Estado Actual del Conocimiento y la Conservación de los Moluscos Continentales de Costa Rica. Revista de Biología Tropical 51(3), 285-292.

Beltran, L. (1985) Algunos Aspectos de la Ecología de una Población del Gastrópodo Marino Littorina ziczac Gmelin (Mesogastropoda: Littorinidae) en una Playa Rocosa del Caribe. (Tesis de Maestria). Universidad de Puerto Rico Piedras. Puerto Rico.

Blanco, J. y F. Scatena (2005) Floods, Habitat Hydraulics and Upstream Migration of Neritina virginea (Gastropoda: Neritidae) in Northeastern Puerto Rico. Caribbean Journal of Science. 41(1), 55-74.

Caicedo, R. (1984) Distribución y Aspectos Taxonómicos de los Moluscos Marinos del Archipiélago de las Mulatas. San Blas (Kuna Yala). (Tesis de Licenciatura). Universidad de Panamá. Panamá.

Caicedo, R. (1989) Distribución y Aspectos Taxonómicos de los Moluscos Marinos del Archipiélago de las Mulatas. Smithsonian Tropical Research Institute. U.S.A.

Capelo, J. y J. Buitrago (1998) Distribución Geográfica de los Moluscos Marinos en el Oriente de Venezuela. Memoria Sociedad de Ciencias Naturales La Salle. Contribución No. 273, Estación de 
Investigaciones Marinas de Margarita Fundación La Salle de Ciencias Naturales 58(150), 109160.

Castillo-Rodríguez, Z. (2014) Biodiversidad de Moluscos Marinos en México. Revista Mexicana de Biodiversidad, Supl. 85, 419-430.

Correa-Sandoval, A. y J. Rodríguez-Castro (2013) Zoogeografía de los Bivalvos Marinos de la Costa de Tamaulipas, México. Revista de Biología Marina y Oceanografía. 48(3), 565-584.

Dance, S. (2002) Smithsonian Handbook Shell. Adorling Kindinly Book. New York. U.S.A.

De La Rosa, J. (1998) Estudio sobre la Distribución de la Malacofauna del Manglar La Ensenada La Claridad, Punta chame con Énfasis en los Géneros Thais y Littorina. (Tesis de Licenciatura). Universidad de Panamá.

Ferney, L. y J. Blanco (2012) Distribución de los Gasterópodos del Manglar, Neritina virgínea (Neritidae) y Littoraria angulifera (Littorinidae) en la Ecorregión Darién, Caribe Colombiano. Rev. Biol. Trop. 60 (1), 219-232.

García, E. (2010) Bocas del Toro Revisited. A Follow-up of Olsson \& McGinty's Report on the Panamanian Archipelago. American Conchologist. 38(2), 4-12.

García, E. (2011) Noteworthy Mollusks from the Gulf of Mexico, Including New Record Sizes and Geographical Extensions. American Conchologist. 39(3), 4-9.

González, L. y A. Navarro (1995) Distribución y Aspectos Taxonómicos de los Gastrópodas Adultos de la Bahía de Achiotes. Distrito de Pedasí. Provincia de Los Santos. (Tesis de Licenciatura). Universidad de Panamá. Panamá.

González, M.; L. Sánchez; Y. González y V. Andrión (2011) Diversidad y Variación Estacional de los Gastropoda en la Playa, La Escucha Provincia de Colón. Informe de Investigación de la V.I.P. Universidad de Panamá.

González, M., L .Sánchez, Y. González, V. Andrión, V. Victoria, J. Gutiérrez, G. Malca, y A. Danzic, (2018). Abundancia y Diversidad de Caracoles (Mollusca: Gastropoda), en la Playa La Escucha, Provincia de Colón. Revista Colón Ciencias, Tecnología y Negocios 5(1), 33-39.

González, M., L .Sánchez, B. Peña, V. Victoria, J. Gutiérrez, K. Anderson, D. Gayle, Gretel Saenz y J. Marscal (2019) Abundancia y Diversidad de Caracoles (Mollusca: Gastropoda) de Piña, Costa Abajo, Provincia de Colón. Revista Colón Ciencias, Tecnología y Negocios 6(2), 13-21.

Hammer, Ø.; Harper, D.A.T.; Ryan; P. D., (2001). PAST: Paleontological Statistics Software Package for Education and Data Analysis. Palaeontologia Electronica 4 (1) [en línea]. Disponible en Web: http://palaeoelectronica.org/2001_1/past/issue1_01.html.

Hurtado, L.A.; M. Frey; P. Gaube; E. Pfeiler and T. K. Marcow (2007) Geographical subdivision, demographic history and gene flow in two sympatric species of intertidal snail, Nerita scabricosta and Nerita funiculata, from the tropical eastern Pacific. Marine Biology 151, 1863-1873. 
Keen, A. (1971) Seashell of Tropical West America. Stanfort University Press. U.S.A. second edition.

Kiat, S. y R. Clements (2008) Taxonomy and Distribution of the Neritidae (Mollusca: Gastropoda) in Singapore. Zoological Studies 47(4), 481-494.

Kiel, S. (2001) Taxonomy and Biogeography of Late Cretaceous Gastropoda. (Tesis de Doctorado). Universidad de Hamburgo, Alemania.

Kohn, A. (1970) Food Habits of the Gastropod Mitra litterata Lamarck: Relation to Trophic Structure of the Intertidal Marine Bench Community in Hawaii. Pacific Science 24, 483-486.

Malaquias, M. A. y D. Reid (2008) Systematic revition of the living species of Bullidae (Mollusca: Gastropoda: Cephalaspidea), with a molecular phylogenetic analysis. Zoological Journal of the Linnean Society 153, 453-543.

McAleece, N., J.D.G. Gage,, P.J.D. Lambshead, G.L.J. Paterson, (1997) BioDiversity Professional Statistics Analysis Software. Jointly developed by the Scottish Association for Marine Science and the Natural History Museum London.

Mazenett-Gutierrez, G.; J. Quintero-Galvis y L. Castro-García (2012) Estructura Poblacional y Variabilidad Fenotípica de Nerita tessellata (Gastropoda: Neritidae) de la Costa Caribe Colombiana de Santa Marta (Magdalena). Rev. Intropica. 7, 21-30.

Miloslavich, P. y E. Huck (2009) Mollusk Assemblages in Seagrasses and Macroalgal Rockyshores in Venezuela: Implementing the NaGISA Protocol. Memoria Sociedad de Ciencias Naturales La Salle. Contribución No. 273, Estación de Investigaciones Marinas de Margarita Fundación La Salle de Ciencias Naturales 171, 81-98.

Moreno, C. (2001). Métodos para Medir la Biodiversidad. Manuales de Tesis SEA. México. D.F. México.

Navarrete, S. (1994) Effects of Interactions Between Predators, Variable Predation Regimes, and Species Body Size on Rocky Intertidal Communities: Comparative and Experimental Approaches. (Doctoral Thesis) Zoology Department Research Funds. Oregon State University. U.S.A.

Nieto-Bernal, R.; L. Chasqui; A. Rodríguez; E. Castro y D. Gil-Agudelo (2013) Composición, Abundancia y Distribución de las Poblaciones de Gasterópodos de Importancia Comercial en La Guajira, Caribe Colombiano. Rev. Biol. Trop. 61 (2), 683-700.

Qin, H. (1995) Polymorphism in Twelve Species of Neritidae (Mollusca: Gastropoda: Prosobranchia) from Hong Kong. (Tesis de Doctorado). Universidad de Hong Kong. China.

Peña, B. (2000). Distribución del Género Nerita en el Distrito de Portobelo, Provincia de Colón. (Tesis de Licenciatura) Escuela de Biología. Centro Regional Universitario de Colón. Universidad de Panamá. Panamá 
Rasmussen, J.; C. Heinberg y E. Håkansson (2005) Planktonic Foraminifer, Bioestratigraphy and the Diachronous Nature of the Lowermost Danian Cerithium Limestone at Stevns Klint, Denmark. Bulletin of the Geological Society of Denmark 52,113-131.

Ríos, K. (2004) Evaluación Del Repoblamiento en el Arrecife de Playa Mora, Jalisco. (Tesis de Licenciatura) Centro Universitario de Ciencias Biológicas y Agropecuarias. Universidad de Guadalajara. México.

Rodríguez, L.; R. Vargas; J. Cortés (2003) Biodiversidad Marina de Costa Rica: Gastrópodos (Mollusca: Gastropoda) de la costa Caribe. Revista de Biología Tropical. 51(3), 305-399.

Solís, G. (2004) Inventario de Moluscos de la Playa La Albina Grande. Guararé. (Tesis de Licenciatura). Universidad de Panamá. Panamá.

Spencer, H.; J. Waters y T. Eichhorst (2007) Taxonomy and nomenclature of black nerites (Gastropoda:Neritimorpha:Nerita) from the South Pacific. Invertebrate Systematics 21, 229-237.

StatSoft Inc. (2004). “Statistica,” Data Analysis Software System, version 7, 